\title{
Antwort auf die Stellungnahme des BAG-Direktors
}

Thierry Carrel ${ }^{a}$, Paul Libera ${ }^{b}$

a Prof. Dr. med., Klinikdirektor Universitätsklinik für Herz- und Gefässchirurgie, Inselspital Bern

b Dr. med., Klinikmanager, Universitätsklinik für Herz- und Gefässchirurgie, Inselspital, Bern
Das BAG will mit seinem jährlichen Qualitätsreport nach eigenem Bekunden zweierlei: Patienten bei der Auswahl des Spitals helfen und Klinikärzte auf Fehler hinweisen, um Verbesserungsprozesse einzuleiten. $\mathrm{Ob}$ es beiden Ansprüchen gerecht wird, kann jeder Klinikarzt für sich entscheiden. Er muss nur den öffentlichen und den vertraulichen BAG-Bericht zu seiner Klinik studieren. Tragischerweise schwächelt die sehr gute Transparenz-Idee des BAG in der Umsetzung, weil aus für diesen Zweck unbrauchbaren sog. vertraulichen Klinikdaten ein offizieller und jedem Bürger zugänglicher Bericht verfasst wird. Fast komödiantische Züge trägt der Statistikteil, den das BAG den Kliniken vertraulich zusendet. Schon der Tagesanzeiger titelte «Zweifelhaftes Spitalranking des BAG» [1]. Ein Spitaldirektor wird zitiert: «Die Onlinedatenbank des BAG (sei) ‘fahrlässig, weil noch nicht ausgereift. Sein Spital schaue die BAG-Statistiken gar nicht an.» «Die vom BAG ausgewiesene Mortalität berücksichtige weder Begleiterkrankungen noch die weiteren Risikofaktoren neben dem Alter
Fehler bei der Kodierung haben bisher nur das Spital und den Kostenträger bilateral tangiert. Spätestens jetzt müssen die offiziellen Kodierrichtlinien überarbeitet werden, wenn «Intraoperativer Ultraschall» und «Kardioplegie» unsinnigerweise als Behandlungsverfahren gelten. Nun also gehen diese eher harmlosen sprachlichen Stilblüten im ICDCHOP-DRG-System (und natürlich auch echte KCodierfehler) in eine offizielle Behörden-Mitteilung ein. Aus kleinen sprachlichen Problemchen bei der Kodierung und Rechnungsstellung im Spital werden gravierende Fehler in einer BAG-Auswertung.

Es ist offensichtlich, dass es Fachbereiche gibt, in denen aus den derzeit vorhandenen Daten keine aussagekräftigen Ergebniskennzahlen abgeleitet werden können. Den kritisierten BAG-Begriff «beteiligte Klinik» sollte man «fallführende Klinik» nennen. Auf die mitunter grotesken BAG-Tabellen «Haupt- und Nebendiagnosen» und «Haupt- und Nebenbehandlungen» würden wir verzichten. Und es wäre besser, die Auswertung für das Jahr X nicht erst im Herbst des

\section{«Echte Handlungsempfehlungen aus einer zwei Jahre alten Qualitätsstatistik abzuleiten, ist schwierig.»}

und dem Geschlecht», so ein Spital-Mediensprecher. Diese Kritik wurde nun in der SÄZ mit Beispielen untermauert [2].

Sicher kann das BAG bei der Angabe der von ihm öffentlich publizierten Spitalsterblichkeit nicht alle Risikofaktoren berücksichtigen. Diese sind nur den Insidern der jeweiligen Fachgebiete bekannt. Wenn aber ein reales Abbild der tatsächlichen Klinikqualität nicht möglich ist, ist es keine Lösung, eine schlechte Methode anzuwenden. Spitäler über Mortalitätsraten bzw. die Relation Sterbefälle/Fallzahl zu vergleichen, ist problematisch. Qualität lässt sich nicht immer in Zahlen messen. So haben maximalversorgende Grossspitäler mit Rund-um die Uhr-Aufnahme oft mehr Hochrisikopatienten zu versorgen als kleinere Spitäler. Dort stirbt man häufiger, weil kränker.
Jahres X plus 2 zu erhalten. Echte Handlungsempfehlungen aus einer zwei Jahre alten Qualitätsstatistik abzuleiten, ist schwierig. Der Blick nach Österreich, wo sich diese Methode der Initiative Qualitätsmedizin erst in der Evaluierungsphase befindet, lohnt vielleicht. In Deutschland wendet nur eine Gruppe von Spitälern, nicht staatlich verordnet, unter sich diese Methode an. In der Schweiz dagegen sollten besser alle Anstrengungen unternommen werden, verlässliche Register innerhalb der einzelnen Fachgesellschaften, gern auch staatlich begleitet, anzulegen.

\section{Literatur}

1 Schmid S. Zweifelhaftes Spitalranking des BAG. tagesanzeiger.ch vom 27.1.2012.

2 Carrel T. Die Suche nach Qualitätsindikatoren für Schweizer Spitäler. Schweiz Ärztezeitung. 2013;94(34):1270 